now manages and carry out the developed guideline at the MDT conference. The aims are to identify challenges and successful initiatives based on individual experiences and to point out areas of development within the MDT conference.

Methodology The study is conducted as a social scientific questionnaire using the complexity theoretic model by Ralph Stacey, designed using MSC (Most Significant Change technique) which focusses on development and dynamics in organizations that are constantly and rapidly changing. 618 participants were identified and invited by either their own MDT leader or by a contact person appointed by the chairman of the cancer group.

Results Answers were calculated using selective coding resulting in themes leading to the following outcome: The MDT conference is a well-established part of clinical practice, and are perceived as significant, qualified and qualifying; Measures that can further qualify the conference decision include more time for the task; A spinoff effect is identified on the conference participants' social and professional relationship, which are developed, valued and supported in relation to the meetings

Conclusion Multiple opportunities for improvement and future development potentials are presented in this article

Disclosures The authors have nothing to disclose. ICMJE forms signed.

Funding and Trial registration

Funding by the Danish Multidisciplinary Cancer Group (DMCG) and the Danish Cancer Collaboration Center (DCCC)

\section{TESTING PREDICTION ACCURACY OF IDEAL AND PROLONGED LENGTH OF HOSPITAL STAY FOLLOWING OVARIAN CANCER CYTOREDUCTION USING MACHINE LEARNING METHODS}

${ }^{1}$ Alexandros Laios, ${ }^{2}$ Camilo De Lelis Medeiros-de-Morais, ${ }^{1}$ Yong Tan, ${ }^{3}$ Gwendolyn Saalmink, ${ }^{1}$ Mohamed Otify, ${ }^{1}$ Amudha Thangavelu, ${ }^{1}$ Richard Hutson, ${ }^{1}$ David Nugent, ${ }^{4}$ Kassio Michell Gomes de Lima, 'George Theophilou. 'St James's University Hospital; Leeds Teaching Hospitals; Gynaecologic Oncology; ${ }^{2}$ School of Pharmacy and Biomedical Science; University of Central Lancashire; ${ }^{3}$ St James's University Hospital; Research and Innovation Centre; ${ }^{4}$ Federal University of Rio Grande Do Norte; Chemistry

\subsection{6/ijgc-2020-ESGO.194}

Introduction/Backgroun Cytoreductive surgery for advanced high grade serous ovarian cancer (HGSOC) patients to achieve complete removal of all visible disease often requires prolonged surgical time and possible multi-visceral resection potentially necessitating HDU support and prolonged hospitalisation. Length of stay (LOS) has been suggested as a marker of quality or effectiveness of short-term care. Identifying modifiable risk factors at admission predicting LOS could lead to appropriately targeted interventions to improve the delivery of care. Modern data mining technologies such as Machine Learning (ML) could be helpful in monitoring hospital stays to improve standards of care. We aimed to improve the accuracy of predicting both ideal and prolonged LOS, by use of ML algorithms.

Methodology A cohort of 176 HGSOC patients, who underwent surgical cytoreduction, from Jan 2014 to Dec 2017 was selected from the ovarian database. They were randomly assigned to 'training' and 'test' subcohorts. ML methods including Linear Discriminant Analysis (LDA), Support Vector Machine (SVM), Decision-Tree-Analysis, and K-Nearest Neighbors were employed to derive predictive information for LOS from selected variables including age, BMI, Surgical Complexity Score (SCS), operative time, preoperative albumin and morbidity score (Clavien-Dindo 3-5). These methods were tested against conventional linear regression. The accepted 'ideal' LOS was deemed to be 5 days or fewer. Prolonged LOS was defined as time spent in the hospital beyond the 90th percentile. Through the introduction of the Enhanced Recovery after Surgery (ERAS) pathway in 2015, effort was made to shorten the LOS for patients following major surgery, whilst still assuring they received effective treatment and highquality care.

Results Mean and median LOS was 4.6 and 4.0 days (IQR $0-38)$, respectively. The delayed LOS group consisted those staying 10 days or longer. The rate of ideal LOS continuously improved for every year from $32 \%$ in 2016 to $73.5 \%$ in 2019 despite increasing mean SCS. For ideal LOS prediction accuracy, ML slightly outperformed conventional logistic regression, with no bowel resection and operative time been the most predictive variables. For prolonged LOS, LDA and SVM were more accurate to predict prolonged LOS than conventional regression. Bowel resection and Clavien-Dindo complications were most importantly contributing to the improved accuracy of the model (figure 1).

Conclusion Predictive ML algorithms may facilitate quality improvement of modern care by improving prediction accuracy for ideal and prolonged LOS. They more accurately identify potentially modifiable factors delaying hospital discharge, which may further inform services performing root cause analysis of LOS.

Disclosures No disclosures.
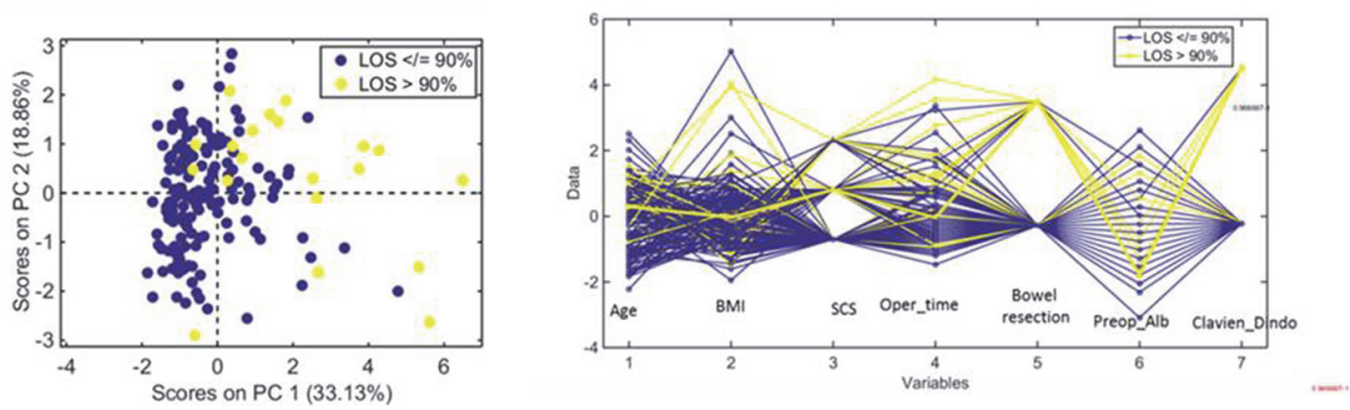

Abstract 334 Figure 1 